CARNETS DE Carnets de géographes

GÉOGRAPHES.

$7 \mid 2014$

Les espaces de l'entre-deux

\title{
Les déguerpissements à Phnom Penh (Cambodge)
}

Déplacements forcés et relocalisations contraintes des citadins pauvres

Julie Blot

C OpenEdition

Journals

Édition électronique

URL : http://journals.openedition.org/cdg/396

DOI : $10.4000 /$ cdg.396

ISSN : 2107-7266

Éditeur

UMR 245 - CESSMA

Référence électronique

Julie Blot, «Les déguerpissements à Phnom Penh (Cambodge) 》, Carnets de géographes [En ligne], 7|

2014, mis en ligne le 01 décembre 2014, consulté le 24 septembre 2020. URL : http://

journals.openedition.org/cdg/396; DOI : https://doi.org/10.4000/cdg.396

(c)

La revue Carnets de géographes est mise à disposition selon les termes de la Licence Creative Commons Attribution - Pas d'Utilisation Commerciale - Pas de Modification 4.0 International. 


\section{LES DEGUERPISSEMENTS A PHNOM PENH (CAMBODGE) DEPLACEMENTS FORCES ET RELOCALISATIONS CONTRAINTES DES CITADINS PAUVRES}

\section{JULIE BLOT}

La forte croissance économique que connaît le Cambodge depuis le début des années 2000 et l'intensification des échanges internationaux entraînent des changements majeurs dans la petite capitale de Phnom Penh, ville secondaire à l'échelle Sud-est asiatique ${ }^{1}$. Jouissant d'une certaine stabilité politique depuis la fin des années 1990, Phnom Penh s'est reconstruit et modernisé, et attire aujourd'hui de plus en plus d'investissements, étrangers et nationaux, notamment dans le secteur immobilier. Les autorités cambodgiennes ont à cœur d'attirer les capitaux étrangers et organisent la "mise en vitrine » de l'espace urbain, selon les modèles consacrés de Singapour ou Bangkok. Ce choix de la compétitivité et de la modernisation conduit à multiplier les déguerpissements ${ }^{2}$ de citadins pauvres, installés sur des terres au statut foncier peu clair, afin de mettre en concession ou de revendre ces terrains ainsi libérés. Les habitants des bidonvilles sont tout particulièrement victimes de cette politique de nettoyage, qui les chasse du centre-ville et les réinstalle sur des sites de relocalisation lointains, ou fait d'eux des « nomades urbains $»^{3}$.

Ma thèse s'est focalisée sur ces déguerpissements qui touchent les quartiers informels phnompenhois, de l'installation des bidonvilles au déplacement forcé des habitants et la reterritorialisation hors-la-ville d'une partie d'entre eux. C'est le processus de changement brutal et involontaire de lieux de vie qui m'intéressait à travers cette étude. Phnom Penh est dans un moment de transition urbaine qui conduit à une certaine violence symbolique et physique vis-à-vis des citadins les plus pauvres qui apparaissent comme des victimes d'un développement dont ils ne profitent pas. J'ai abordé cette problématique à différentes échelles en me demandant quelles sont les conséquences de ces déguerpissements sur les populations réinstallées. Quel est l'impact de la création ex nihilo de ces «nouveaux villages » sur la périphérie rurale de Phnom Penh ? Et comment les déguerpissements inscrivent-ils dans l'espace et dans le paysage une ségrégation socio-spatiale croissante? Dit autrement, comment refaire territoire après une déterritorialisation forcée ?

La méthodologie adoptée pour cette étude a eu pour objectif de rendre les discours et perceptions des citadins chassés de la ville ou menacés de l'être mais aussi d'analyser l'évolution spatiale de la périphérie phnompenhoise sous l'effet des réinstallations. J'ai travaillé dans divers bidonvilles du centre et sites de relocalisation de la périphérie suivant un questionnaire semi-directif sur des indicateurs socio-économiques, prolongé par des

\footnotetext{
${ }^{1}$ Franck, M., Goldblum, Ch., Taillard, C., 2012, Territoires de l'urbain en Asie du Sud-Est. Métropolisation en mode mineur, CNRS éditions, Paris, 308 p.

${ }^{2}$ Le terme «déguerpissement» est plus usité en Afrique francophone, il désigne le déplacement forcé de citadins par les forces de l'ordre au motif que leur installation est illégal du point de vue des autorités. Le terme souligne la précipitation, la violence et le dénigrement vécu par les populations qui le subissent, c'est pourquoi il est ici préféré au mot «éviction » plus neutre et général.

${ }^{3}$ Dupont, V., 2010, « Création de nomades urbains et appauvrissement. Impact des politiques d'éradication des camps de squatters à Delhi », Revue Tiers Monde, 201, pp. 25-45
} 
conversations plus libres sur le passé des bidonvillois ou des déguerpis et sur leurs aspirations d'avenir. Cette thèse se situe donc à la rencontre de plusieurs thématiques de géographie humaine : la pauvreté urbaine et les déplacements forcés. Elle cherche à nourrir le débat de la reinstallation forcée et la question de la justice spatiale, et conduit ainsi à s'interroger sur le « droit à la ville » pour les citadins pauvres.

\section{Déterritorialisation et reterritorialisation des bidonvillois}

Le site de relocalisation est un espace singulier, il dénote dans le paysage, il se démarque et ne peut être défini ni comme rural ni comme urbain. Son plan orthogonal aux parcelles régulières le distingue des villages voisins, qui se présente comme un village-rue traditionnel. Mes enquêtes dans ces villages ont montré des reterritorialisations diverses et sélectives. Si certains sites ont disparu purement et simplement, où que seules quelques maisons demeurent au milieu de villages fantômes, la plupart des sites de relocalisation ont perduré, signe qu'ils répondaient à l'attente d'une partie des déguerpis, prêts à accepter cette compensation plutôt que de tout perdre. Dès lors la reterritorlisation est acceptée et se réalise à travers divers type d'appropriation du site (construction du logement, implantation de nouveaux géosymboles, appropriation des espaces collectifs) qui se mue peu à peu en village ou en quartier.

\section{La réinstallation contrainte : une injustice spatiale ?}

Cette dialectique s'exprime dans les discours des déplacés et des citadins menacés de déguerpissement. Plusieurs niveaux de lecture peuvent éclairer le déguerpissement : une justification légale (statut foncier, utilité publique) ; un argument d'ordre moral faisant valoir que le déplacement est entrepris au bénéfice des habitants (insalubrité, criminalité, dangerosité) ; et des motifs tus (transaction financière, éloignement des indésirables...). Les registres de l'illégalité et l'immoralité ont tendance à gommer la question de justice ${ }^{4}$. À l'inverse, en recueillant les propos des déguerpis, on constate qu'une injustice spatiale est exprimée, qui n'est pas un état de fait mais le résultat d'un processus. Parler d'injustice et de justice c'est exprimer un sentiment vécu par les populations qui subissent le déplacement. En passant par l'appréhension de l'avant et de l'après-éviction j'ai voulu éclairer ce vécu et le rapport à l'espace qu'il implique, notamment par la comparaison qu'établissent les déplacés «avant, c'était mieux », exprimant une certaine nostalgie mais surtout la dégradation bien réelle des conditions d'existence d'une majeure partie d'entre eux.

\section{Quel droit à la ville pour les citadins pauvres ?}

L'observation des lieux de départ avant et après le déguerpissement permet de confronter les discours à la pratique et de constater les effets des politiques d'aménagement. Si au début des années 1990 les évictions servaient surtout à libérer des terrains pour réaménager une ville en mauvais état, avec une valeur croissante du foncier depuis 2000 la plupart des anciens grands bidonvilles ont laissé place à des projets immobiliers privés comme le montre bien l'exemple de Koh Pich, ancien îlot maraîcher transformé en quartier de luxe. C'est en cela que le « droit à la ville », notion éminemment politique, se pose. Quelle ville construit-on ? Une ville débrassée de ses pauvre, d'après les propos d'un enquêtés qui considéraient qu'à Phnom Penh «ils font une ville pour les riches et une ville pour les pauvres ». En effet, le processus de déguerpissement-relocalisation a cela de particulier qu'il ne vise pas seulement à déplacer des populations, mais aussi à favoriser la réimplantation et la stabilisation des populations pauvres en périphérie. Cette intention est clairement ressentie par les déplacée.

\footnotetext{
${ }^{4}$ BLOT, J., SPIRE, A., 2014, « Déguerpissements et conflits autour des légitimités citadines dans les villes du Sud », [in] L’Espace Politique [en ligne], n²2, URL : http://espacepolitique.revues.org/2893
} 
Le bilan des déguerpissements laisse entrevoir une évolution dangereuse pour l'avenir de la capitale cambodgienne, dont les autorités continuent de décider ou d'encourager le déplacement progressif des poches de pauvreté vers des zones de plus en plus éloignées et isolées. Ce mode de gestion des quartiers informels est le moins juste socialement et le plus risqué politiquement. La relocalisation apparaît plus comme un moyen de s'accaparer des terres au profit d'une élite économique proche du pouvoir, plutôt que comme une façon de régler le problème des bidonvilles par le haut. Actuellement, cette politique est de moins en moins acceptée, les protestations s'expriment de plus en plus, sous les formes les plus diverses (manifestations, mobilisation des réseaux sociaux, flashmob'...) et dirigées par des leaders charismatiques (tels que Tep Vanny et Yorm Bopha). La lutte anti-éviction rejoint de nombreux mécontentements et les activistes de la lutte anti éviction ont massivement rejoint l'opposition lors des élections de juillet 2013 qui ont été un véritable séisme pour le PPC, parti au pouvoir depuis plus de trente ans. L'instabilité politique dans laquelle est entrée le pays depuis lors donne une nouvelle tribune à ces mouvements.

\section{Relocalisation des bidonvilles dans la périphérie de Phnom Penh}

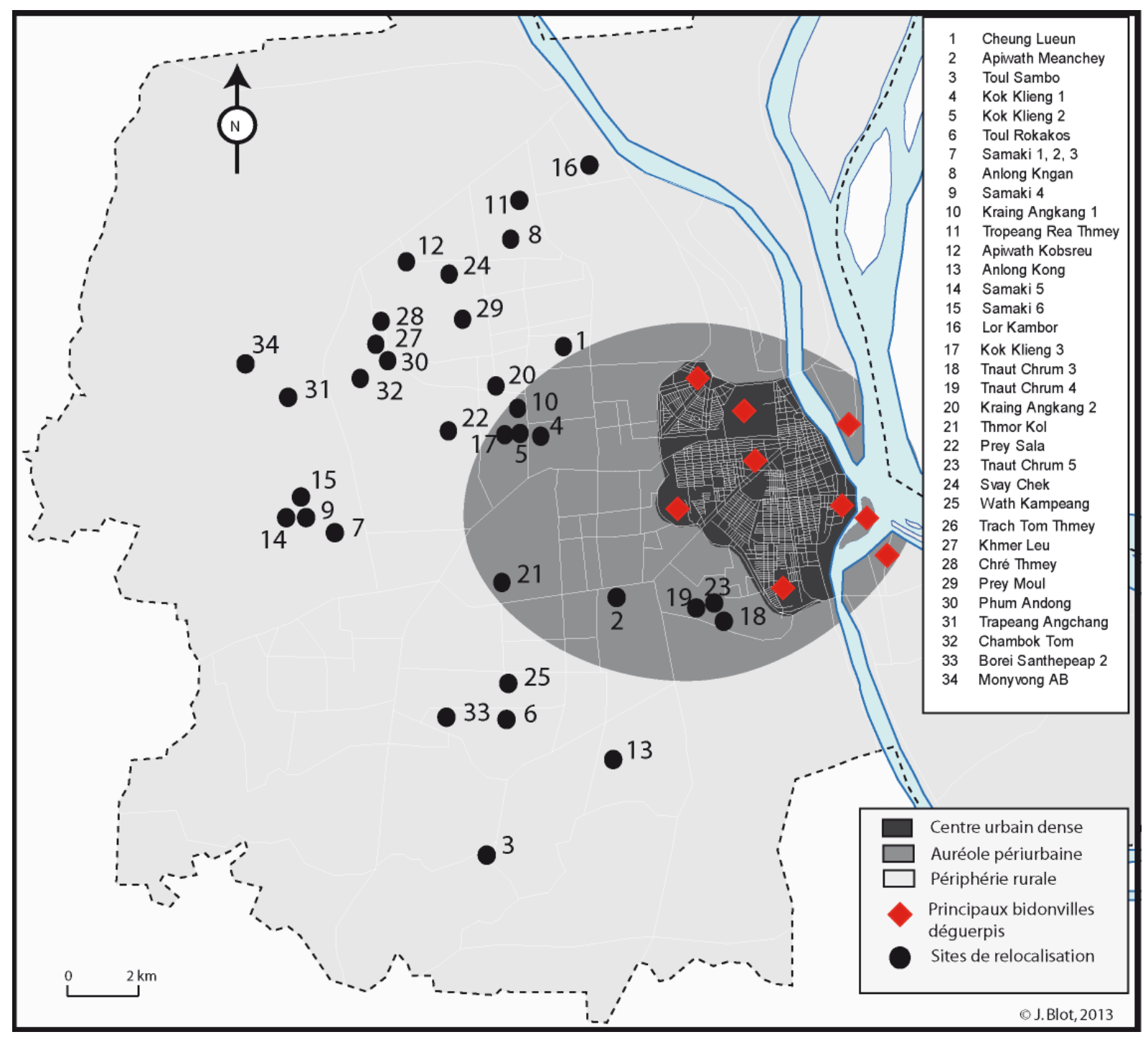


Discipline

Géographie

$\underline{\text { Directeurs }}$

Pr. Olivier Sevin, Université Paris IV-Sorbonne, laboratoire ENeC (UMR 8185)

Université

Université Paris IV-Sorbonne

Membres du jury de thèse soutenue le 4 décembre 2013

M. Michel ANTELME, Professeur, INALCO (président du jury)

M. Charles GOLDBLUM, Professeur émérite, Université Paris 8 Vincennes-Saint Denis (rapporteur)

M. Christian BOUQUET, Professeur émérite, Université Bordeaux III

Mme Véronique LASSAILLY-JACOB, Professeur émérite, Université de Poitiers (rapporteur)

M. Olivier SEVIN, Professeur, Université Paris IV-Sorbonne (directeur de thèse)

$\underline{\text { Situation professionnelle actuelle }}$

Chargée de cours à l'INALCO et post-doctorante à l'IRD

Contact de l'auteur

julie.blot@voila.fr 